\title{
Identification of Molecular Distinctions Between Normal Breast-Associated Fibroblasts and Breast Cancer-Associated Fibroblasts
}

\author{
Andrea Sadlonova • Damon B. Bowe • Zdenek Novak • \\ Shibani Mukherjee • Virginia E. Duncan • \\ Grier P. Page • Andra R. Frost
}

Received: 15 August 2008 / Accepted: 24 November 2008 /Published online: 18 March 2009

(C) The Author(s) 2008. This article is published with open access at Springerlink.com

\begin{abstract}
Stromal fibroblasts influence the behavior of breast epithelial cells. Fibroblasts derived from normal breast (NAF) inhibit epithelial growth, whereas fibroblasts from breast carcinomas (CAF) have less growth inhibitory capacity and can promote epithelial growth. We sought to identify molecules that are differentially expressed in NAF versus $\mathrm{CAF}$ and potentially responsible for their different growth regulatory abilities. To determine the contribution of soluble molecules to fibroblast-epithelial interactions, NAF were grown in $3 \mathrm{D}$, transwell or direct contact co-cultures with
\end{abstract}

Electronic supplementary material The online version of this article (doi:10.1007/s12307-008-0017-0) contains supplementary material, which is available to authorized users.

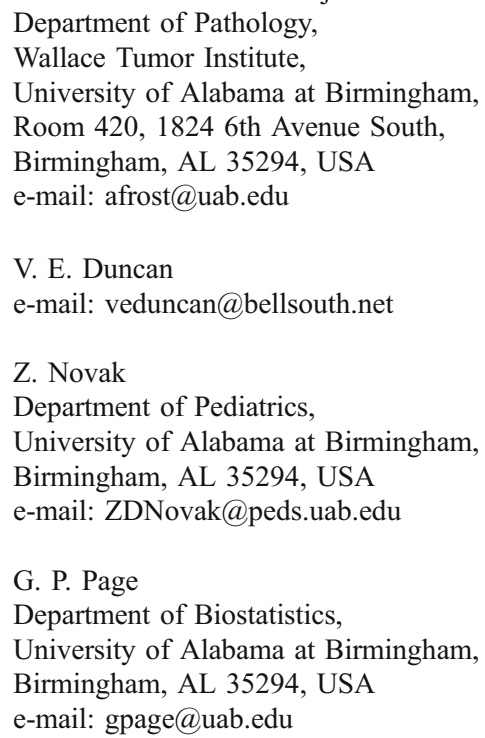

MCF10AT epithelial cells. NAF suppressed proliferation of MCF10AT in both direct contact and transwell co-cultures, but this suppression was significantly greater in direct cocultures, indicating involvement of both soluble and contact factors. Gene expression profiling of early passage fibroblast cultures identified 420 genes that were differentially expressed in NAF versus $\mathrm{CAF}$. Of the eight genes selected for validation by real-time PCR, FIBULIN 1, was overexpressed in NAF, and DICKKOPF 1, NEUREGULIN 1, PLASMINOGEN ACTIVATOR INHIBITOR 2, and TISSUE PLASMINOGEN

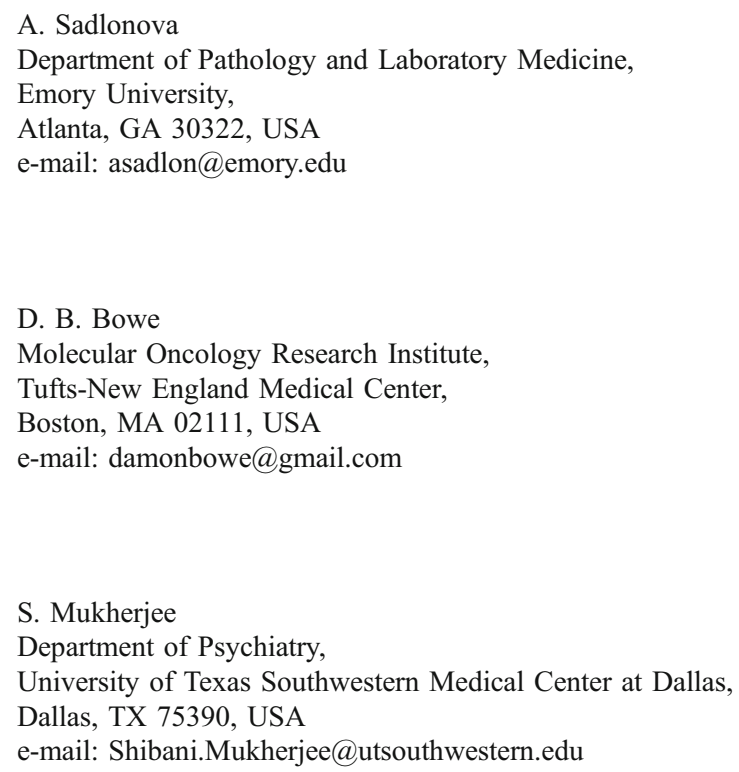


ACTIVATOR were overexpressed in CAF. A higher expression of FIBULIN 1 in normal- than cancer-associated fibroblastic stroma was confirmed by immunohistochemistry of breast tissues. Among breast cancers, stromal expression of Fibulin 1 protein was higher in estrogen receptor $\alpha$ positive cancers and low stromal expression of Fibulin 1 correlated with a higher proliferation of cancer epithelial cells. In conclusion, expression profiling of NAF and CAF cultures identified many genes with potential relevance to fibroblast-epithelial interactions in breast cancer. Furthermore, these early passage fibroblast cultures can be representative of gene expression in stromal fibroblasts in vivo.

Keywords Breast cancer - Fibroblasts · Fibulin 1 . Gene expression profiling $\cdot$ Stroma

$\begin{array}{ll}\text { Abbreviations } \\ \text { 3D } & \text { Three dimensional } \\ \text { BrdU } & \text { Bromodeoxyuridine } \\ \text { CAF } & \text { Carcinoma-associated fibroblasts } \\ \text { DKK1 } & \text { DICKKOPF 1 } \\ \text { ECM } & \text { Extracellular matrix } \\ \text { FBLN1 } & \text { FIBULIN 1 } \\ \text { FITC } & \text { Fluorescein isothiocyanate } \\ \text { MMP1 } & \text { MATRIX METALLOPROTEINASE 1 } \\ \text { NAF } & \text { Fibroblasts derived from normal breast } \\ \text { NRG1 } & \text { NEUREGULIN 1 } \\ \text { PAI2 } & \text { PLASMINOGEN ACTIVATOR INHIBITOR } 2 \\ \text { PLAT } & \text { TISSUE PLASMINOGEN ACTIVATOR } \\ \text { QRT } & \text { Quantitative real-time PCR } \\ \text { THBS3 } & \text { THROMBOSPONDIN 3 } \\ \text { TFPI2 } & \text { TISSUE FACTOR PATHWAY INHIBITOR 2 }\end{array}$

\section{Introduction}

Breast tumorigenesis is a multifaceted process involving molecular and functional alterations in both the stromal and epithelial compartments of the breast. The interaction between these two compartments is important in the tumorigenic process and is rooted in a complex network of molecules belonging to families of growth factors, immunomodulatory factors, steroid hormones, and extracellular matrix (ECM) components and proteases [1-3]. Several studies indicate that stromal fibroblasts surrounding normal and cancerous breast epithelium exert a modulatory effect on the epithelium, the nature of which is dependent upon the state of the fibroblasts and the epithelium [3-5]. Specifically, stromal fibroblasts in normal breast serve a protective function and exert inhibitory signals on the growth of normal epithelium, while cancer-associated stromal fibroblasts act more permissively and allow or promote growth of normal and cancer epithelium. In vitro studies with normal-breast associated fibroblasts (NAF) demonstrate that NAF inhibit the growth of the nontumorigenic breast epithelial cell line, MCF10A, and its more transformed, tumorigenic derivative, $\operatorname{MCF} 10 \mathrm{AT}[3,5]$. In vivo, admixed NAF exert an inhibitory effect on histologically normal epithelium but also limit cancer development and growth as shown in the MCF10AT xenograft model of proliferative breast disease [6].

Conversely, fibroblasts derived from breast cancer tissues (CAF) possess permissive or promoting abilities for epithelial cell growth both in vitro and in vivo and exhibit molecular and functional characteristics similar to that of activated stromal fibroblasts normally associated with wound healing [3, 4]. In contrast to NAF, CAF proliferate at a higher rate and secrete increased levels of growth factors, ECM proteins and immunomodulatory factors [2, 7-9]. The ability of CAF to modulate epithelial cell growth is dependent on the phenotype of the corresponding epithelium. As has been previously shown, CAF inhibit the growth of the MCF10A cells in vitro [3] but promote the growth of breast cancer cell lines, such as MCF-7, in vitro and in vivo $[4,10,11]$. Therefore, the biologic effect of CAF is influenced by the molecular and functional properties of the CAF and the responsiveness of the epithelial cells. Only a few specific molecules derived from CAF, such as Stromal Derived Factor 1 and Hepatocyte Growth Factor, have been shown to contribute to the tumorigenic process $[4,12]$. Given the complexity of these stromal-epithelial interactions and the molecular heterogeneity of breast cancers, there are likely many more fibroblast-derived molecules important in breast carcinogenesis and cancer progression that remain to be identified.

In this work, we identify genes that are differentially expressed in NAF and CAF. These gene products may be associated with a growth inhibitory function of normal breast stroma and a growth permissive or promoting function of breast cancer stroma. Our data also indicate that fibroblast-epithelial interactions involve both insoluble and soluble secreted molecules. Insoluble molecules may be embedded in the ECM or located on cell membranes. Using gene expression profiling and quantitative RT-PCR, we identified multiple genes, encoding both soluble and matrix-bound molecules, that are differentially expressed in in vitro cultures of NAF and CAF and that are associated with remodeling of the ECM and/or are secreted proteins that affect the growth of epithelial cells. Additionally, our data confirm that the differential expression of the ECM glycoprotein Fibulin 1 (FBLN1) in NAF and CAF cultures recapitulated expression of FBLN1 in the fibroblastic stroma of histologically normal breast and breast cancer tissues. 


\section{Materials and Methods}

Maintenance of Epithelial Cell Lines and Fibroblasts

MCF10AT cells (Karmanos Cancer Institute, Detroit, Michigan) were cultivated in Dulbecco's Modified Eagle's Medium/Ham's F-12 (Cambrex, Walkersville, MD) supplemented with $0.1 \mu \mathrm{g} / \mathrm{ml}$ cholera toxin (Calbiochem, San Diego, CA), $10 \mu \mathrm{g} / \mathrm{ml}$ insulin (Sigma, St. Louis, MO), $0.5 \mu \mathrm{g} / \mathrm{ml}$ hydrocortisone (Sigma), $0.02 \mu \mathrm{g} / \mathrm{ml}$ epidermal growth factor (Upstate Biotechnology, Lake Placid, NY) and $5 \%$ horse serum (Invitrogen, Carlsbad, CA) in a humidified, $5 \% \mathrm{CO}_{2}, 37^{\circ} \mathrm{C}$ incubator.

Human breast fibroblasts from mammoplasties and breast cancer resections were isolated and characterized by immunocytochemistry as per Sadlonova et al. [3]. Fibroblasts were subjected to immunocytochemical evaluation with antivimentin (mouse IgG1, clone V9; Neomarkers, Fremont, CA, USA), anti-epithelial membrane antigen (mouse IgG2a, clone ZCE113; Zymed, San Francisco, CA, USA), and anticytokeratin (CK) 5/CK 8 (mouse IgG1, clone C-50; Neomarkers) as confirmation of their stromal origin (i.e. strong vimentin expression, and absence of epithelial membrane antigen and CK 5/CK 8). Fibroblasts were cultured in Dulbecco's Modified Eagle's Medium supplemented with $10 \%$ fetal bovine serum.

\section{Oligonucleotide Microarray Hybridization and Analysis}

RNA was isolated from subconfluent cultures, passages 24, of two NAF (isolated by us from two different individuals) and three CAF (two cultures isolated by us from two different individuals and the Hs574T cell line, a CAF purchased from the American Type Culture Collection (Manassas, VA)) using TRIzol $^{\circledR}$ reagent (Invitrogen). Biotinylated cRNA probes were generated from the isolated RNA and hybridized individually to high-density oligonucleotide microarrays (Hu95A array, Affymetrix, Santa Clara, CA). Hybridization was detected using a streptavidin-phycoerythrin conjugate and quantified with a high-resolution scanner.

\section{RNA Isolation and Real-Time PCR}

RNA was isolated from eight NAF and seven CAF cultures (all isolated by us from different individuals), passages 3-6, followed by RNA clean-up with RNeasy ${ }^{\circledR}$ Minikit columns (Qiagen, Valencia, CA). All RNA samples were subjected to DNase pretreatment prior to cDNA synthesis. RNA was converted into double stranded cDNA using the HighCapacity cDNA Archive kit (Applied Biosystems, Foster City, CA). Primer/probe sets for DICKKOPF 1 (DKK1), FIBULIN 1 (FBLN1), MATRIX METALLOPROTEINASE 1 (MMP1), NEUREGULIN 1 (NRG1), PLASMINOGEN
ACTIVATOR-INHIBITOR 2 (PAI2), THROMBOSPONDIN 3 (THBS3), TISSUE PLASMINOGEN ACTIVATOR (PLAT), and TISSUE FACTOR PATHWAY INHIBITOR 2 (TFPI2) (TaqMan ${ }^{\circledR}$ Gene Expression Assays-on-Demand ${ }^{\mathrm{TM}}$, Applied Biosystems, Foster City, CA) interrogated the following sequences: DKK1 - Hs00183740_m1, reference sequence NM_012242; FBLN1-Hs00242545_m1, reference sequences NM_001996, NM_006487, NM_006486, NM_006485; FBLN1C-Hs00242546_m1, reference sequences NM_001996; FBLN1D-Hs00972628_m1, reference sequence NM_006486; MMP1-Hs00233958_m1, reference sequence NM_002421; NRG1-Hs00247620_m1, reference sequences NM_004495, NM_013958, NM_013957, NM_013956, NM_013964, NM_013962, NM_013961, NM_013960; PAI2-Hs00234032_m1, reference sequence NM_002575; PLAT_Hs00263492_m1, reference sequences NM_033011, NM_000931, NM_000930; THBS3Hs00200157_m1, reference sequence NM_007112; TFPI2Hs00197918_m1, reference sequence NM_006528.

Sequences for the ribosomal S9 primer/probe set follow: F5' ATCCGCCAGCGCCATA 3', R-5' TCAATGTGCT TCTGGGAATCC 3', probe-5' 6FAMAGCAGGTGGTGAA CATCCCGTCCTTTAMRA 3'.

Each culture was assayed in triplicate and each reaction contained $1 \mu \mathrm{l}$ cDNA, $12.5 \mu \mathrm{l} 2 \times$ TaqMan ${ }^{\circledR}$ Universal PCR Master Mix (Applied Biosystems), $1.25 \mu 1$ TaqMan ${ }^{\circledR}$ Gene Expression Assays-on-Demand ${ }^{\mathrm{TM}}$ primer/probe set for each target. Fluorescent signal data was collected by the ABI Prism 7700 Sequence Detection System. Ribosomal S9 was used as the internal reference and was selected because it exhibits minimal variability in tissues of different origins [13]. The standard curve method was employed to determine relative expression levels of each gene.

Measuring Proliferation of MCF10AT Cells Grown with Fibroblasts in 3D Direct and Transwell Co-cultures

In 3D direct and transwell co-cultures, the ratio of epithelial cells to fibroblasts was 2:1. Cells were grown in serum free medium and plated on a layer of Growth-Factor-Reduced Matrigel (BD Biosciences, Franklin Lakes, NJ), as previously described [3]. For 3D direct cultures, cells were grown in eight-well chamber slides following the protocol in Sadlonova et al. [3] For transwell experiments, MCF10AT cells and fibroblasts were grown in separate compartments with the epithelial cells plated in the Matrigel-coated well and the fibroblasts in the Matrigelcoated insert $(0.4 \mu \mathrm{M}$ pore size, polyester, Corning Costar, Lowell, MA). Cultures were incubated in a $37^{\circ} \mathrm{C}, 5 \% \mathrm{CO}_{2}$ humidified incubator for 14 days. To label proliferating cells, $0.2 \mathrm{mg} / \mathrm{ml}$ bromodeoxyuridine (BrdU) was applied to all cultures for $24 \mathrm{~h}$. BrdU-labeled cells were counted by flow cytometry, as previously described [3]. Briefly, 
MCF10AT cells were stained with fluorescein isothiocyanate (FITC)-conjugated anti-BrdU (mouse $\mathrm{IgG}_{1}$, clone B44, BD Biosciences Immunocytometry Systems). In direct cocultures, MCF10AT cells were distinguished from fibroblasts by labeling with an allophycocyanin-conjugated antiEpCAM (mouse IgG1, clone EBA-1; BD Biosciences Immunocytometry Systems). Negative controls included staining with FITC-conjugated $\operatorname{IgG}_{1}$ (mouse $\operatorname{IgG}_{1}, \mathrm{~K}$ isotype control, BD Biosciences Pharmingen). Cells were analyzed on a BD FACS Calibur ${ }^{\mathrm{TM}}$ flow cytometer (BD Biosciences), and the percentage of BrdU-FITC positive MCF10AT cells was calculated.

Immunohistochemistry for FBLN1, Estrogen Receptor and $\mathrm{Ki}-67$

Formalin-fixed, paraffin-embedded breast cancers $(n=35)$, corresponding uninvolved breast tissue $(n=32)$ and tissue from breast reduction specimens $(n=7)$ were obtained from the archives of the University of Alabama at Birmingham Department of Pathology and clinical information was obtained from the Department of Surgery after Institutional Review Board Approval. Our methods of performing immunohistochemistry have been reported in the literature [14-17]. For estrogen receptor (ER) and Ki-67 staining, sections ( $5 \mu \mathrm{m}$ thick) were subjected to low temperature antigen retrieval with enzymatic pretreatment, which consists of pre-digestion in $0.1 \%$ trypsin (Type II-S from porcine pancreas, Sigma Chemicals, St. Louis, MO) in phosphate buffered saline for $15 \mathrm{~min}$ in a $37^{\circ} \mathrm{C}$ oven followed by incubation in $10 \mathrm{mM}$ citrate buffer, $\mathrm{pH} 6$, for $0 \mathrm{~h}$ at $80^{\circ} \mathrm{C}$, as previously described [14]. Sections for FBLN1 staining did not require antigen retrieval. All sections were incubated with an aqueous solution of $3 \%$ hydrogen peroxide for $5 \mathrm{~min}$ followed by incubation with $1 \%$ goat serum. Sections were incubated with two monoclonal antibodies to FLBN1 (clone B-5, Santa Cruz Biotechnology, Santa Cruz, CA at $1 \mu \mathrm{g} / \mathrm{ml}$ or clone A311, from the laboratory of Scott Argraves [18], at $1 \mu \mathrm{g} / \mathrm{ml}$ ), a monoclonal antibody to ER $\alpha$ (clone ER88, Biogenex, San Ramon, CA, at 1:30 dilution $(0.33 \mathrm{mg} / \mathrm{ml}$ total protein $)$ ) or a monoclonal antibody to Ki-67 (clone MIB-1, Biogenex, San Ramon, CA, at $1: 30$ dilution $(0.37 \mathrm{mg} / \mathrm{ml}$ total protein)) diluted in phosphate buffered saline ( $\mathrm{pH} 7.6)$ containing $1 \%$ bovine serum albumin, $1 \mathrm{mM}$ ethylenediamine tetraacetic acid, and $1.5 \mathrm{mM}$ sodium azide for one hour at room temperature. This was followed by secondary detection with a streptavidin horseradish peroxidase system (Signet Laboratories) and diaminobenzidine was utilized as the chromogen. Negative control slides, without addition of primary antibody, were also prepared.

All immunohistochemical stains were examined and scored by two of the authors (ARF and AS) concurrently.
To semi-quantify FBLN1 immunostaining, a scoring system based on both staining intensity and percentage of cells or area stained was utilized, as previously described [14, $15,17]$. In this system, the intensity of staining is graded from 0 (no staining) to 4 (greatest staining possible). The proportion of cells/area staining at each intensity is multiplied by the corresponding intensity value and these products are added to obtain an immunostaining score (immunoscore) ranging from 0 to 4 . For ER $\alpha$ and Ki-67, the percentage of cancer epithelial cells with nuclear staining was quantified.

\section{Statistical Analysis}

Microarray array images were processed to extract expression quantification with MAS 5 using the Affymetrix GCOS software. High-Dimensional-Biology-Statistics (HDBStat!; Department of Biostatistics, University of Alabama at Birmingham [19]) was used for analysis of the gene expression data, including quantile-quantile normalization, quality control and comparison of gene expression. Genes determined to be differentially expressed and chosen for validation had a fold difference of at least 2.5 and a $p$ value $\leq$ 0.05 by the equal variance $t$ test. The percentage of BrdU and Ki-67 positive cells, real-time PCR expression values and tumor size were compared by the $t$ test for unequal variances. The proportion of patients with positive lymph nodes in FBLN1 low versus high breast cancers was compared using Fisher's exact test. Immunohistochemical scores for FBLN1 were compared by the Wilcoxon signed rank two sample test or the Mann Whitney test, as appropriate.

\section{Results}

Gene Expression Profiling of NAF and CAF Revealed Many Differentially Expressed Genes

We have previously shown that NAF have a greater ability to inhibit epithelial cell growth than CAF in direct contact co-cultures [3]. To identify molecules through which NAF may inhibit epithelial growth to a greater extent than $\mathrm{CAF}$, the gene expression profiles of NAF and CAF were compared. Affymetrix Hu95A arrays interrogating approximately 10,000 full length genes were used to compare gene expression. Early passage NAF (two cultures) and CAF (three cultures) were used. Each of the fibroblast cultures were from a different individual. The comparison of mean expression in NAF versus CAF yielded 420 genes that were differentially expressed with a $p$ value $\leq 0.05$ and at least a 2.5 -fold difference in expression level. Of the 420 differentially expressed genes, 180 were overexpressed in NAF 
and 240 overexpressed in CAF (Supplemental Tables 1 and 2).

NAF Suppressed Proliferation of MCF10AT Epithelial Cells Through Soluble and Insoluble Factors

To assist us in selecting genes identified as differentially expressed by expression microarray for validation, we wanted to know if both soluble and insoluble secreted factors were important in the growth inhibition of epithelial cells induced by NAF. To determine this, we prepared 3D transwell and direct co-cultures of MCF10AT epithelial cells and NAF. Transwell co-cultures allow assessment of soluble secreted molecules that can traverse the filter to influence cells in a paracrine manner. In direct co-cultures, the roles of both soluble secreted molecules and insoluble molecules, such as matrix- or membrane-bound molecules that depend on direct contact between cells or between cells and the ECM, can be analyzed. In transwell co-cultures, the mean percentage of MCF10AT cells labeled by BrdU (i.e., BrdU labeling index) was decreased by $20 \%$ in co-culture with NAF $(p=0.011)$. The NAF utilized were derived from three different individuals. In direct co-cultures, the mean reduction in BrdU labeling by the same three NAF was $46 \%(p<0.001)$ (Fig. 1). There was variability among the three NAF in their ability to inhibit proliferation of MCF10AT, particularly in direct contact cocultures. The greater reduction in proliferation of MCF10AT in direct versus transwell co-culture was significant $(p=0.04)$ (Fig. 1). These results indicate that inhibition of epithelial growth by NAF is mediated by a mixture of direct-contact/ insoluble and soluble factors. Therefore, we selected differentially expressed genes from the microarray analysis encoding both soluble and matrix-bound, insoluble molecules for validation by quantitative, real-time PCR (QRT).

\section{Expression of a Subset of Differentially Expressed Genes was Confirmed by Real-Time PCR}

We selected eight genes from the list of 420 differentially expressed genes in NAF and CAF for validation by QRT (Fig. 2a, Supplemental Tables 1 and 2). The primary criterion for selecting genes for validation was that they encoded a secreted protein, either soluble or matrix-bound, that was known to regulate cell growth, migration, invasion and/or ECM remodeling. Among those selected, some genes had a high fold expression ratio (PAI2 and TFPI2 with a 51-52-fold CAF/NAF expression), a medium fold ratio (DKK1 and MMP1 with a 9-10 fold CAF/NAF expression), and a lower fold ratio (NRG1 and PLAT with a 4-fold CAF/NAF expression; FBLN1 and THBS3 with a 45 fold NAF/CAF expression). For validation by QRT analysis, early passage NAF and CAF derived from eight and seven different individuals, respectively, were used.

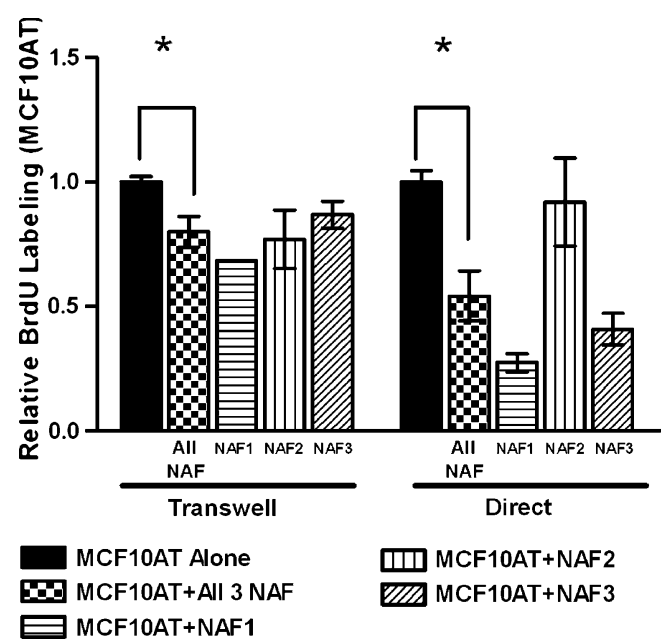

Fig. 1 Proliferation of MCF10AT in 3D direct and transwell cocultures with NAF. Direct and transwell 3D (i.e., in Matrigel) cocultures of MCF10AT cells with each of three NAF from different individuals were prepared. BrdU labeling of MCF10AT cells was counted by flow cytometry. Each NAF (i.e., NAF1, NAF2 and NAF3) suppressed proliferation of co-cultured MCF10AT cells to some extent in transwell co-cultures, and two of the three NAF (i.e., NAF1 and NAF3) suppressed proliferation of MCF10AT in direct co-cultures. When comparing the overall reduction in proliferation of MCF10AT induced by the three NAF in all transwell co-cultures combined $(n=$ 10, checkered bar) to MCF10AT grown without co-cultured NAF (black bar), the decrease in proliferation was significant $(p=0.011)$. Similarly, the overall decrease in proliferation induced by the three NAF in all direct co-cultures combined $(n=14$, checkered bar) compared to MCF10AT monocultures (black bar) was significant $(p<0.001)$. However, the degree of suppression was significantly greater in direct than transwell co-cultures $(p=0.04)$. Data are normalized to corresponding MCF10AT monocultures. Mean and standard error are shown

Two genes overexpressed in NAF cultures were selected for validation: the ECM protein FBLN1 (5.4 fold greater, $p=0.011)$ and the ECM glycoprotein THBS3 (4.1 fold greater, $p=0.014$ ) (Fig. 2a and Supplemental Table 1). Of these two genes, FBLN1 expression was confirmed to be higher among NAF cultures compared to CAF cultures by QRT (Fig. 2b). No difference in expression was detected between NAF and CAF for THBS3 (Fig. 2b).

Six genes overexpressed in CAF were selected for validation: the Wnt antagonist DKK1 (9.8 fold greater, $p=$ 0.002), MMP1 (10.3 fold greater, $p=0.016$ ), NRG1 (4.1 fold greater, $p=0.010$ ), TFPI2 (51.5 fold greater, $p=0.001$ ), which is involved in the regulation of coagulation, and two members of the plasminogen activating/plasmin system-PAI2 (also known as SERPINB2, 52.2 fold greater, $p=0.015$ ) and PLAT (also known as tPA, 4.2 fold greater, $p=0.041$ ) (Fig. 2a and Supplemental Table 1). In the QRT validation analysis, the expressilon of DKK1, NRG1, PAI2, and PLAT was confirmed to be higher in CAF cultures $(p<0.05)$ (Fig. 2b). The expression of MMP1 was also found to be higher in CAF than NAF, but this difference reached only borderline 
Fig. 2 Results of expression array analysis and QRT of genes selected for validation. a Graphical presentation of expression array data for the eight significantly $(p<0.05)$ differentially expressed genes selected for QRT validation. Mean expression of two NAF and three $\mathrm{CAF}$ cultures is presented relative to the expression in NAF (NAF expression=1). b Expression of selected genes as assessed by QRT in eight NAF and seven CAF cultures. Mean expression and standard deviation are presented relative to expression in NAF. Significant differences in expression in $\mathrm{NAF}$ and CAF were found for FBLN1 $(p<0.001)$, DKK1 $(p=$ $0.033)$, NRG1 $(p=0.043)$, PAI2 $(p=0.002)$, and PLAT $(p=$ 0.037 ), indicated by asterisks
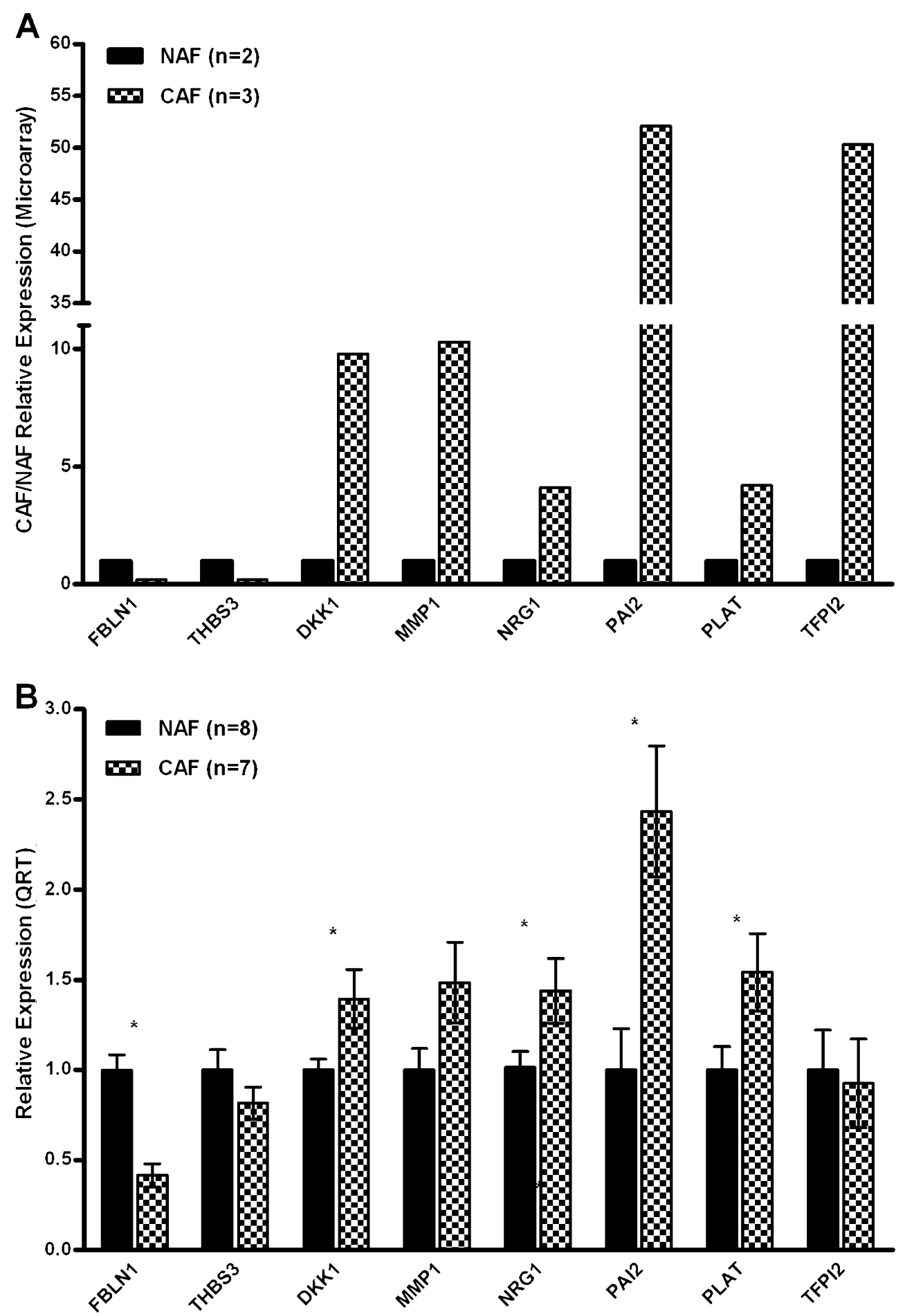

statistical significance ( $p=0.065)$ (Fig. $2 b$ ). There was no difference in expression of TFPI2 in NAF and CAF. Therefore, FBLN1, DKK1, NRG1, PAI2, and PLAT were confirmed to be differentially expressed in NAF and CAF by QRT.

Expression of FBLN1 Was Reduced in Breast Cancer Stroma

To identify genes differentially expressed in NAF and CAF, we used in vitro cultures of fibroblasts isolated from breast tissues. We used early passages of these cells in an attempt to reduce changes in gene expression induced by cell culture. However, gene expression can differ in vitro and in vivo. To determine whether any of the expression differences in NAF and CAF identified in vitro are reflective of expression in breast tissues ex vivo, expression of FBLN1 in the fibroblastic stroma (fibroblasts and ECM) surrounding breast epithelium was assessed by immunohistochemistry in cancers and corresponding benign, histologically 
Fig. 3 Immunostaining for FBLN1. a FBLN1 expression by immunohistochemistry with either A311 or B-5 antibody is lower in the stroma of breast cancers $(n=32)$ than in the stroma of corresponding benign breast $(n=32)$ from the same individual $(p<0.001$ and $p=0.047$ for antibodies A311 or B-5, respectively). Expression in the stroma of benign breast (from breast cancer resection specimens) and in normal breast (from mammoplasty specimens) $(n=7)$ is similar. Expression of FBLN1 is greater in cancer epithelium than benign epithelium with the A311 antibody $(p=0.002)$. The mean immunoscore and standard error are shown. b Immunohistochemical staining of one breast cancer and corresponding benign breast demonstrating greater staining of stroma $(S)$ (both extracellular matrix and fibroblasts) surrounding epithelial structures in benign breast than in breast cancer. In this particular case, immunostaining is greater in cancer epithelium $(E)$ than in benign epithelium with both antibodies. (bar $=50 \mu \mathrm{m})$
A

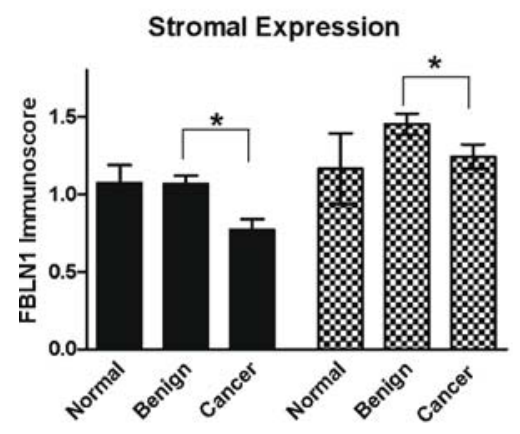

Antibody A311 Antibody B-5
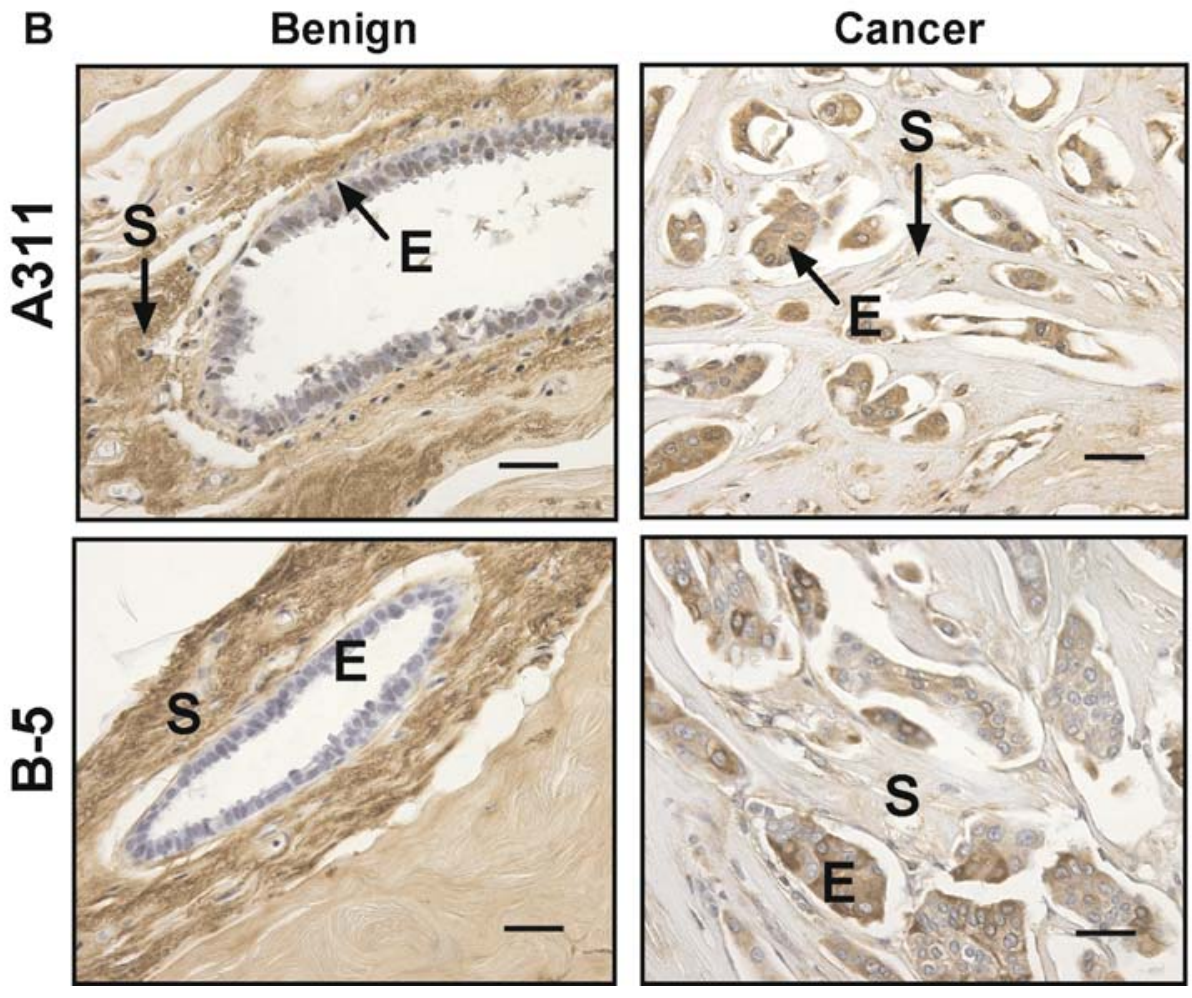

normal breast from breast cancer resection specimens and in normal breast tissue from breast reduction specimens (Fig. 3b). We selected FBLN1 for further validation because (1) it has been reported to suppress the growth and motility cancer cells [20-22], (2) the fold difference in expression between NAF and CAF was relatively high (Fig. 2b), and (3) antibodies suitable for use in formalinfixed, paraffin-embedded tissues were readily available. Two different monoclonal antibodies to FBLN1 were used, A311 and B-5. Both antibodies identify all documented splice variants and recognize epitopes located at the $\mathrm{N}$ terminus of FBLN1 protein [23].
Thirty-two breast cancers and corresponding uninvolved, histologically normal tissue (i.e., benign) from the same breast cancer resection specimen, as well as tissue from seven breast reduction specimens (i.e., normal) were stained with both anti-FBLN1 antibodies. The histologic sections of benign breast selected for analysis were derived from an area of the breast not immediately adjacent to the breast cancers. The immunostaining was semi-quantified using a scoring system that combines the number of cells or area stained and the intensity of the staining. This scoring system has been used by us and others previously [14-17]. Stroma surrounding histologically normal breast epithelium 
Table 1 Stromal immunoscores for FBLN1 in 32 matching pairs of benign breast and breast cancer

\begin{tabular}{|c|c|c|c|c|c|c|c|}
\hline \multirow[t]{3}{*}{ Benign/cancer pair } & \multicolumn{3}{|c|}{ Antibody A311 } & \multirow[t]{3}{*}{ Benign/cancer pair } & \multicolumn{3}{|c|}{ Antibody B-5 } \\
\hline & \multicolumn{3}{|c|}{ Stromal immunoscore } & & \multicolumn{3}{|c|}{ Stromal immunoscore } \\
\hline & Benign & Cancer & Fold difference $^{a}$ & & Benign & Cancer & Fold difference \\
\hline $\mathrm{A}$ & 0.53 & 0.04 & 13.13 & A & 1.00 & 0.18 & 5.71 \\
\hline $\mathrm{B}$ & 1.00 & 0.13 & 7.69 & $\mathrm{C}$ & 1.80 & 0.63 & 2.88 \\
\hline $\mathrm{C}$ & 1.15 & 0.18 & 6.27 & B & 1.50 & 0.65 & 2.31 \\
\hline $\mathrm{D}$ & 1.18 & 0.33 & 3.62 & G & 1.60 & 0.85 & 1.88 \\
\hline $\mathrm{E}$ & 1.24 & 0.47 & 2.64 & $\mathrm{P}$ & 1.55 & 0.83 & 1.88 \\
\hline $\mathrm{F}$ & 1.75 & 0.70 & 2.50 & $\mathrm{~S}$ & 2.20 & 1.40 & 1.57 \\
\hline G & 1.05 & 0.43 & 2.47 & I & 1.80 & 1.15 & 1.57 \\
\hline $\mathrm{H}$ & 1.10 & 0.50 & 2.20 & $\mathrm{~V}$ & 1.60 & 1.08 & 1.49 \\
\hline $\mathrm{I}$ & 1.35 & 0.63 & 2.16 & $\mathrm{~F}$ & 1.60 & 1.13 & 1.42 \\
\hline $\mathrm{J}$ & 0.76 & 0.36 & 2.10 & $\mathrm{~J}$ & 1.46 & 1.06 & 1.38 \\
\hline $\mathrm{K}$ & 0.96 & 0.48 & 2.02 & $\mathrm{~N}$ & 1.90 & 1.40 & 1.36 \\
\hline $\mathrm{L}$ & 1.50 & 0.75 & 2.00 & Q & 1.50 & 1.13 & 1.33 \\
\hline $\mathrm{M}$ & 1.21 & 0.71 & 1.70 & $\mathrm{H}$ & 1.10 & 0.85 & 1.29 \\
\hline $\mathrm{N}$ & 1.23 & 0.83 & 1.48 & $\mathrm{D}$ & 1.35 & 1.05 & 1.29 \\
\hline $\mathrm{O}$ & 1.70 & 1.15 & 1.48 & $\mathrm{O}$ & 1.48 & 1.15 & 1.28 \\
\hline $\mathrm{P}$ & 0.95 & 0.65 & 1.46 & $\mathrm{~T}$ & 1.60 & 1.25 & 1.28 \\
\hline Q & 1.35 & 0.93 & 1.46 & Z & 1.88 & 1.50 & 1.25 \\
\hline $\mathrm{R}$ & 0.85 & 0.60 & 1.42 & E & 0.85 & 0.75 & 1.13 \\
\hline $\mathrm{S}$ & 1.30 & 0.93 & 1.41 & $\mathrm{BB}$ & 1.28 & 1.13 & 1.13 \\
\hline $\mathrm{T}$ & 1.25 & 0.93 & 1.35 & $\mathrm{M}$ & 1.40 & 1.27 & 1.11 \\
\hline $\mathrm{U}$ & 1.13 & 0.90 & 1.25 & $\mathrm{~L}$ & 2.33 & 2.33 & 1.00 \\
\hline $\mathrm{V}$ & 0.90 & 0.80 & 1.13 & $\mathrm{R}$ & 1.35 & 1.40 & 0.96 \\
\hline W & 1.05 & 0.99 & 1.07 & W & 1.73 & 1.85 & 0.93 \\
\hline$X$ & 1.08 & 1.05 & 1.02 & $X$ & 1.45 & 1.60 & 0.91 \\
\hline $\mathrm{Y}$ & 0.53 & 0.53 & 1.00 & $\mathrm{U}$ & 1.48 & 1.65 & 0.89 \\
\hline $\mathrm{Z}$ & 1.03 & 1.05 & 0.98 & $\mathrm{CC}$ & 1.60 & 1.90 & 0.84 \\
\hline $\mathrm{AA}$ & 1.00 & 1.23 & 0.82 & $\mathrm{DD}$ & 1.20 & 1.45 & 0.83 \\
\hline $\mathrm{BB}$ & 0.71 & 0.98 & 0.72 & $\mathrm{AA}$ & 1.40 & 1.80 & 0.78 \\
\hline $\mathrm{CC}$ & 0.95 & 1.35 & 0.70 & $\mathrm{Y}$ & 0.75 & 1.00 & 0.75 \\
\hline $\mathrm{DD}$ & 0.93 & 1.35 & 0.69 & $\mathrm{FF}$ & 0.80 & 1.08 & 0.74 \\
\hline $\mathrm{EE}$ & 0.93 & 1.65 & 0.56 & $\mathrm{EE}$ & 1.35 & 2.05 & 0.66 \\
\hline $\mathrm{FF}$ & 0.59 & 1.15 & 0.51 & K & 0.65 & 1.25 & 0.52 \\
\hline
\end{tabular}

${ }^{\mathrm{a}}$ Benign/Cancer

and within breast cancers was immunoscored. The mean immunoscore for FBLN1 was significantly higher in stromal fibroblasts and associated ECM in benign breast than in cancer-associated stromal fibroblasts and ECM when using either antibody A311 $(p=0.001)$ or antibody B$5(p=0.047)$ (Fig. 3a). Of the 32 breast cancer and benign tissue pairs, FBLN1 expression was higher in benign stroma than cancer-associated stroma in $75 \%$ and $63 \%$ of cases immunostained with antibody A311 and antibody B-5, respectively (Table 1). Because the NAF cultures were derived from the breasts of women without cancer, we also assessed expression of FBLN1 in breast reduction specimens. The mean immunoscore was similar in the fibroblastic stroma of the normal breast in reduction specimens and the benign tissue from breast cancer patients, stained with either antibody (Fig. 3a). These results indicate that expression of
FBLN1 is reduced in CAF in vitro and in the fibroblastic stroma of breast cancer ex vivo.

We also noted that the cytoplasm of epithelial cells in some breast cancers stained more strongly than the epithelium in the histologically normal counterpart. The normal or benign epithelium did not stain with the B-5 antibody, whereas there was cytoplasmic staining of epithelium using the A311 antibody (Fig. 3b). With the A311 antibody, the mean immunoscore of the benign ductolobular epithelium in cancer resection specimens was 3 -fold lower than in the corresponding cancer epithelium $(p=0.002)$. A similar result with the A311 antibody was previously reported [18]. The epithelium of five breast cancers stained with the B-5 antibody, but there was no significant difference in staining between benign and cancer epithelium ( $p=0.082)$ (Fig. 3a). 


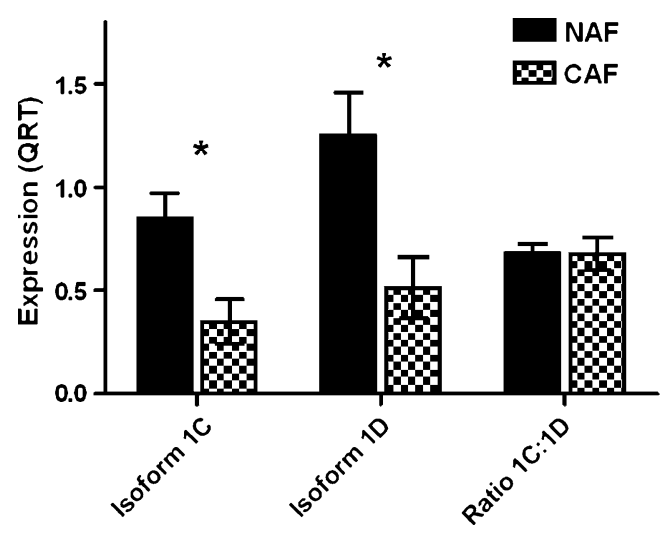

Fig. 4 Expression of FBLN1 isoforms in NAF and CAF cultures. Expression of FBLN1C and FBLN1D was assessed by QRT using isoform-specific primer/probe sets in all eight NAF and seven CAF. Expression of FBLN1C and FBLN1D was lower in CAF than NAF ( $p=0.008$ and $p=0.011$, respectively, marked by asterisks). Furthermore, the ratio of FBLN1C to FBLN1D did not differ in NAF and $\mathrm{CAF}$. The mean and standard deviation are shown

The Ratio of Expression of FBLN1 Isoforms did not Differ in NAF and CAF Cultures

There are four different splice variants of FBLN1 named A, $\mathrm{B}, \mathrm{C}$ and D. Only FBLN1C and FBLN1D have been detected in breast cancer cell lines and tissues [18]. Furthermore, it has been reported that the ratio of mRNA expression of FBLN1C to FBLN1D is increased in breast cancers in comparison to corresponding normal breast [24]. Additionally, expression of FBLN1C is induced by estrogen [25], which suggests a potential mechanism for the increased expression of FBLN1C in breast cancer. To determine whether there was a similar increase in the ratio of FBLN1C to 1D in CAF compared to NAF, we assessed expression of FBLN1C and FBLN1D in the NAF and CAF cultures by QRT. Expression of both FBLN1C and FBLN1D isoforms was significantly lower in CAF than NAF ( $p=0.008$ and $p=0.011$, respectively), and the ratio of

Table 2 Breast cancer clinicopathologic data

\begin{tabular}{ll}
\hline Age (years) & $27-83$ \\
Race (\%) & \\
White & 73 \\
African American & 24 \\
Other & 3 \\
Tumor size (cm) & $1.1-12.0$ \\
Lymph node status (\%) & \\
Positive & 49 \\
Negative & 40 \\
Unknown & 11 \\
Pathologic stage (\%) & \\
I-II & 57 \\
III-IV & 29 \\
Unknown & 14 \\
\hline
\end{tabular}

1C to $1 \mathrm{D}$ was similar in NAF and CAF (Fig. 4). Because all FBLN1 antibodies available recognized both fibulin isoforms, we were unable to compare isoform expression in the stroma of the breast tissues by immunohistochemistry.

Expression of FBLN1 is Higher in Estrogen Receptor-Positive than Estrogen Receptor-Negative Carcinomas

Because expression of FBLN1C is induced by estrogen through estrogen receptor (ER) $\alpha[23,24]$, we determined whether expression of FBLN1 differed in ER $\alpha$-positive versus -negative carcinomas. Thirty-five breast cancers (the 32 cancers with corresponding normal breast plus three additional cancers without corresponding normal breast) were divided into $E R \alpha$-positive and -negative subtypes, based on a the percentage of cells with nuclei that stained for $\mathrm{ER} \alpha$ (i.e., less than $10 \%=\mathrm{ER} \alpha$ negative). Clinical and pathologic information related to these 35 cancers is summarized in Table 2. The immunoscores for FBLN1 were compared between ER $\alpha$-positive and -negative carcinomas. Using the A311 antibody, FBLN1 in the stroma was significantly higher in ER $\alpha$-positive than -negative cancers $(p=0.032$, Fig. 5). The mean FBLN1 immunoscore in cancer stroma with the B-5 antibody was also higher in ER $\alpha$-positive cancers, but this did not reach statistical significance $(p=0.097)$. Similarly, the mean FBLN1 immunoscore in cancer epithelium with either the A311 or B-5 antibody was higher in ER $\alpha$-positive cancers, but this was not statistically significant ( $p=0.307$ and $p=0.167$, respectively) (Fig. 5). These findings further support an associa-

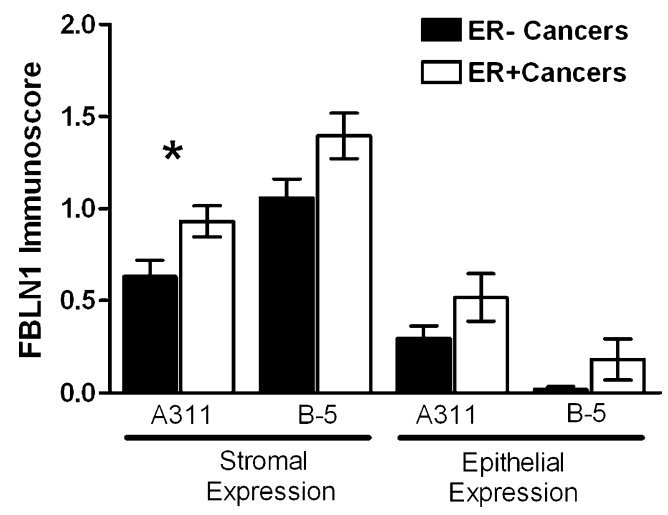

Fig. 5 Comparison of FBLN1 immunoscores in ER $\alpha$-positive and negative breast cancers. FBLN1 expression was assessed by immunohistochemistry in 35 breast cancers. Nineteen were ER $\alpha$-negative, 14 were ER $\alpha$-positive and the ER status was unknown in two. Expression of FBLN1 was higher in the fibroblastic stroma of ER $\alpha$ positive cancers than ER $\alpha$-negative cancers, but this was statistically significant with antibody A311 ( $p=0.032)$ only. The difference in FBLN1 expression in the epithelium of ER $\alpha$-positive versus -negative cancers was not statistically significant. The mean immunoscore and standard error are presented 
tion between FBLN1 expression, particularly in the stroma, and the presence of ER $\alpha$ in cancer epithelial cells.

Higher Expression of FBLN1 in Fibroblastic Stroma is Associated with Lower Rates of Cancer Proliferation

FBLN1 has been demonstrated to inhibit in vitro adhesion and motility of various cancer cell lines, including breast cancer $[20,21]$, and to suppress the growth of human fibrosarcoma cells [22]. Therefore, its loss in breast cancer stroma may allow enhanced growth and invasion of cancer cells. We compared proliferation of cancer epithelial cells in breast cancers with higher versus lower expression of FBLN1 in both stroma and epithelium. The mean FBLN1 immunoscore for each antibody in cancer stroma or epithelium was used as the corresponding cut-off value for higher versus lower expression. Proliferation was determined by immunohistochemistry for Ki-67. In general, the rate of proliferation (i.e., the percentage of epithelial cells labeled by Ki-67) was lower in breast cancers with higher stromal FBLN1 expression (Fig. 6a). However, this difference was only statistically significant for stromal FBLN1 assessed with the A311 antibody $(p=0.034)$, but not with the B-5 antibody $(p=0.178)$ and not for epithelial FBLN1 with either antibody (A311, $p=0.468$; B-5, $p=$ 0.173 ). To determine whether there was any correlation between FBLN1 expression in breast cancers and other indicators of invasiveness and growth (i.e., tumor size and lymph node metastasis) of the breast cancers, we compared these parameters in cancers with higher versus lower FBLN1 immunoscores in stroma or epithelium with both antibodies. There was no significant difference in tumor size or the percentage of patients with lymph node metastases in FBLN1 higher versus FBLN1 lower (stromal or epithelial expression) cancers (Fig. 6b,c).

\section{Discussion}

The vast array of molecules involved in breast stromalepithelial interactions makes it difficult to identify dominant molecules affecting breast cancer initiation and progression. The ambiguity of the spatial and temporal origin of carcinogenesis-related functional and molecular alterations adds another layer of complexity. Even though these alterations have been identified in both stromal and epithelial compartments early in the carcinogenic process [26-28], it is still unclear which compartment is affected first - the epithelium, stroma or both of them simultaneously. These complex issues emphasize a need for additional assessment of the molecular and functional signatures of fibroblasts in normal and cancerous tissues that can eventually expand our understanding of the role of fibroblast-epithelial interactions in cancer.

Results from the current study complement our previous work demonstrating that NAF have a greater inhibitory effect on the proliferation of breast epithelial cells than CAF [3]. We now show that both soluble and matrix- or membrane-bound molecules are important for the inhibitory signal. The greater inhibition of epithelial growth by NAF in direct co-cultures is likely a result of the closer proximity of epithelial cells and fibroblasts allowing for direct contact between different cell types and their ECM. However, significant inhibition of epithelial cell growth by NAF in
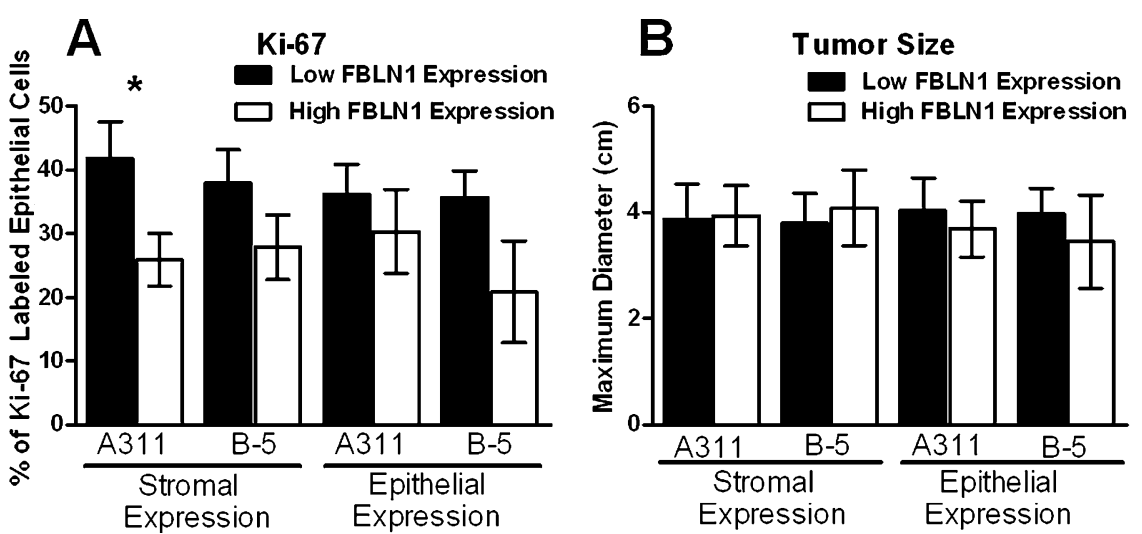

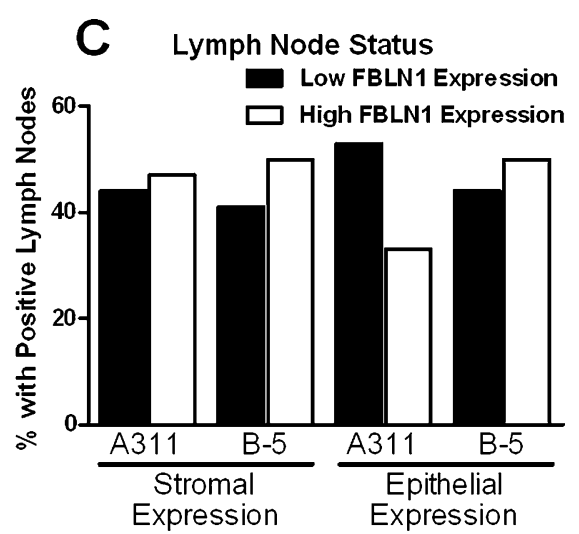

Fig. 6 Proliferation, tumor size, and lymph node status in breast cancers with lower versus higher FBLN1 expression. Thirty-five breast cancers were assessed for FBLN1 expression by immunohistochemistry using antibody A311 or B-5. Cancers were divided into lower versus higher FBLN1 expression in stroma or epithelium based on the mean immunoscore for stromal or epithelial expression with each antibody (i.e., mean FBLN1 immunoscore was 0.74 for stromal expression with A311, 1.19 for stromal expression with B-5, 0.37 for epithelial expression with A311, and 0.08 for epithelial expression with B-5) (as in Fig. 3). a Proliferation, as measured by Ki-67 labeling of cancer epithelial cells, was lower in cancers with higher stromal expression of FBLN1, but this was statistically significant only with the A311 antibody $(p=0.034)$. There was no significant difference in proliferation in cancer with higher versus lower epithelial expression of FBLN1. b, c There was no difference in tumor size (b) or the percentage of patients with positive lymph nodes (c) in breast cancers with higher versus lower stromal or epithelial FBLN1 
transwell cultures indicates that soluble secreted factors are also important. Therefore, our selection of differentially expressed genes for validation included soluble secreted factors, ECM-bound proteins and molecules that contribute to remodeling of the ECM.

Remodeling of the ECM is characteristic of the stromal response to cancer, contributes to the tumor microenvironment and results in molecular alterations that affect cancer behavior $[29,30]$. In CAF, we observed significant overexpression of several molecules involved in ECM remodeling-PAI2 and PLAT. PAI2 inhibits ECM remodeling by inhibiting urokinase plasminogen activator (uPA) [31-33], while PLAT activates a variety of proteins embedded in the ECM by cleaving plasminogen to plasmin and thereby promoting tissue degeneration and ECM remodeling [34, 35]. Overexpression of TFPI2 in CAF was not confirmed by QRT, but TFPI2 is an inhibitor of coagulation and is proposed to be a maintenance factor of ECM remodeling [36]. Our results indicate a borderline increase in MMP1. MMP1 breaks down collagens and other ECM components and has been reported to be expressed at a higher level in breast cancers, but primarily in cancer epithelial cells rather than stromal fibroblasts [37]. However, stromal-derived MMP-1 has been shown to increase breast cancer cell migration and invasion by cleaving protease-activated receptor 1 [38].

Of the two ECM proteins chosen for validation (FBLN1 and THBS3), only FBLN1 was found to be differentially expressed. FBLN1 inhibits in vitro adhesion and motility of various carcinoma cell lines [20]. THBS3 was recently detected in a small number of breast tumors [39, 40]. However, the function of THBS3 is not well defined and this is the first account of THBS3 expression in breast fibroblasts.

Each of the soluble secreted factors chosen for validation, DKK1 and NRG1, were found to be differentially expressed. The Wnt signaling pathway contributes to mammary gland development and tumorigenesis [41]. DKK1 is an antagonist of Wnt signaling and may play an anti-tumorigenic role [42]. However, expression of DKK1 was recently found to be increased in breast cancer cell lines with the ability to metastasize to bone and in the serum of breast cancer patients with bone metastasis [43]. NRG1 is an EGF-like signaling molecule that binds to transmembrane tyrosine kinase receptors of the ErbB family and governs the ductal differentiation of the mammary epithelium. Recent studies demonstrated that it was capable of activating the ErbB2 oncoprotein in breast cancer cells, and NRG1 overexpression in transgenic mice lead to increased breast tumor formation [44, 45]. Therefore, overexpression of these secreted molecules by CAF may enhance breast cancer epithelial cell growth and metastasis.

The extent to which the gene expression profiles of in vitro cultured fibroblasts reflect their gene expression in vivo is not well defined. It is likely that components of the molecular signatures of NAF and CAF are lost during the isolation process and growth in vitro. However, it has been found that the expression of some molecules, such as SMA, in myofibroblasts remains unchanged after multiple subcultures [4]. This persistence of expression may be specific only to some molecules, while for others, expression is more context-dependent and changes when placed in vitro. We demonstrated that expression of one gene, FBLN1, was higher in NAF than CAF cultures in vitro and, correspondingly, in stromal fibroblasts and their ECM in normal breast than in breast cancer ex vivo. Therefore, in vitro breast fibroblast cultures can accurately represent expression of some molecules in stromal fibroblasts of the breast in vivo.

We did not find an increase in the ratio of FBLN1C to FBLN1D in NAF and CAF, as has been reported for breast cancers in general [24]. Because FBLN1C expression is induced by estrogen through ER $\alpha$ [24], the overexpression of FBLN1C in breast cancers may be limited to the ER $\alpha$ expressing epithelial component, rather than the stroma. $\mathrm{ER} \alpha$ has only rarely been detected in adult stromal fibroblasts of the breast [46], and this expression is not detectable by immunohistochemistry [47]. Interestingly, we did find a correlation between the stromal expression of FBLN1 and ER $\alpha$ in cancer epithelial cells, suggesting that paracrine factors produced by $\mathrm{ER} \alpha$ positive epithelial cells may increase FBLN1 expression in stromal fibroblasts.

FBLN1 reduces the adhesion and motility of breast cancer cells in vitro and the growth of fibrosarcomas in a mouse xenograft model [20-22]. Therefore, decreased FBLN1 in breast cancer stroma may provide a microenvironment that is more conducive to epithelial cell growth and migration than stroma in normal breast. In support of this possibility, cancers with higher FBLN1 in breast stroma had a lower rate of epithelial proliferation than did cancers with lower stromal FBLN1. This relationship is confounded by the lower rate of proliferation of ER $\alpha$-positive carcinomas [15]. In the 35 breast cancers studied here, the percentage of Ki-67 labeled cells was $46 \%$ in the ER $\alpha$ negative cancers compared to $16 \%$ in the ER $\alpha$-positive cancers. The observed increase in epithelial proliferation in cancers with lower stromal FBLN1, however, did not correlate with the clinical data in our study in that there were no differences in tumor size or lymph node status in breast cancers with lower versus higher stromal expression of FBLN1. As has been previously described [18], epithelial expression of FBLN1, as assessed with the A311 antibody, was significantly greater in breast cancers than in normal epithelium in our study.

Acknowledgements We thank Dr. Scott Argraves for supplying the Fibulin 1 antibody A311. This work was supported by the National Cancer Institute (R03CA10595 and R03CA97472), the Department of 
Defense Breast Cancer Research Program (DAMD17-03-10514) and the American Cancer Society (RSG-05-207-01-TBE).

Open Access This article is distributed under the terms of the Creative Commons Attribution Noncommercial License which permits any noncommercial use, distribution, and reproduction in any medium, provided the original author(s) and source are credited.

\section{References}

1. Radisky ES, Radisky DC (2007) Stromal induction of breast cancer: inflammation and invasion. Rev Endocr Metab Disord 8:279-287

2. Tlsty TD, Coussens LM (2006) Tumor stroma and regulation of cancer development. Annu Rev Pathol 1:119-150

3. Sadlonova A, Novak Z, Johnson MR et al (2005) Breast fibroblasts modulate epithelial cell proliferation in three-dimensional in vitro co-culture. Breast Cancer Res 7:R46-59

4. Orimo A, Gupta PB, Sgroi DC et al (2005) Stromal fibroblasts present in invasive human breast carcinomas promote tumor growth and angiogenesis through elevated SDF-1/CXCL12 secretion. Cell 121:335-348

5. Shekhar MP, Werdell J, Santner SJ, Pauley RJ, Tait L (2001) Breast stroma plays a dominant regulatory role in breast epithelial growth and differentiation: implications for tumor development and progression. Cancer Res 61:1320-1326

6. Sadlonova A, Mukherjee S, Bowe DB et al (2007) Human breast fibroblasts inhibit growth of the MCF10AT xenograft model of proliferative breast disease. Am J Pathol 170:1064-1076

7. Tan TT, Coussens LM (2007) Humoral immunity, inflammation and cancer. Curr Opin Immunol 19:209-216

8. Balkwill F (2004) Cancer and the chemokine network. Nat Rev Cancer 4:540-550

9. Hawsawi NM, Ghebeh H, Hendrayani SF et al (2008) Breast carcinoma-associated fibroblasts and their counterparts display neoplastic-specific changes. Cancer Res 68:2717-2725

10. Koyama H, Kobayashi N, Harada M et al (2008) Significance of tumor-associated stroma in promotion of intratumoral lymphangiogenesis: pivotal role of a hyaluronan-rich tumor microenvironment. Am J Pathol 172:179-193

11. Sasser AK, Mundy BL, Smith KM et al (2007) Human bone marrow stromal cells enhance breast cancer cell growth rates in a cell line-dependent manner when evaluated in 3D tumor environments. Cancer Lett 254:255-264

12. Kuperwasser C, Chavarria T, Wu M et al (2004) From the cover: reconstruction of functionally normal and malignant human breast tissues in mice. Proc Natl Acad Sci U S A 101:4966-4971

13. Blanquicett C, Johnson MR, Heslin M, Diasio RB (2002) Housekeeping gene variability in normal and carcinomatous colorectal and liver tissues: applications in pharmacogenomic gene expression studies. Anal Biochem 303:209-214

14. Frost AR, Sparks D, Grizzle WE (2000) Methods of antigen recovery vary in their usefulness in unmasking specific antigens in immunohistochemistry. Appl Immunohistochem Mol Morphol 8:236-243

15. Talley LI, Grizzle WE, Waterbor JW, Brown D, Weiss H, Frost AR (2002) Hormone receptors and proliferation in breast carcinomas of equivalent histologic grades in pre- and postmenopausal women. Int J Cancer 98:118-127

16. Chhieng DC, Tabbara SO, Marley EF, Talley LI, Frost AR (2003) Microvessel density and vascular endothelial growth factor expression in infiltrating lobular mammary carcinoma. Breast $\mathrm{J}$ 9:200-207
17. Grizzle WE, Myers RB, Manne U, Stockard CR, Harkins LE, Srivastava S (1998) Factors affecting immunohistochemical evaluation of biomarker expression in neoplasia. In: Hanausek M, Walaszek Z (eds) John Walker's methods in molecular medicine-tumor marker protocols. Humana, Totowa, NJ, pp 161-179

18. Pupa SM, Argraves WS, Forti S et al (2004) Immunological and pathobiological roles of fibulin-1 in breast cancer. Oncogene 23:2153-2160

19. Trivedi P, Edwards JW, Wang J et al (2005) HDBStat!: a platformindependent software suite for statistical analysis of high dimensional biology data. BMC Bioinformatics 6:86

20. Twal WO, Czirok A, Hegedus B et al (2001) Fibulin-1 suppression of fibronectin-regulated cell adhesion and motility. $\mathrm{J}$ Cell Sci 114:4587-4598

21. Hayashido Y, Lucas A, Rougeot C, Godyna S, Argraves WS, Rochefort H (1998) Estradiol and fibulin-1 inhibit motility of human ovarian- and breast-cancer cells induced by fibronectin. Int J Cancer 75:654-658

22. Qing J, Maher VM, Tran H, Argraves WS, Dunstan RW, McCormick JJ (1997) Suppression of anchorage-independent growth and matrigel invasion and delayed tumor formation by elevated expression of fibulin-1D in human fibrosarcoma-derived cell lines. Oncogene 15:2159-2168

23. Greene LM, Twal WO, Duffy MJ et al (2003) Elevated expression and altered processing of fibulin-1 protein in human breast cancer. Br J Cancer 88:871-878

24. Bardin A, Moll F, Margueron R et al (2005) Transcriptional and posttranscriptional regulation of fibulin-1 by estrogens leads to differential induction of messenger ribonucleic acid variants in ovarian and breast cancer cells. Endocrinology 146:760-768

25. Moll F, Katsaros D, Lazennec G et al (2002) Estrogen induction and overexpression of fibulin-1C mRNA in ovarian cancer cells. Oncogene 21:1097-1107

26. Moinfar F, Man YG, Arnould L, Bratthauer GL, Ratschek M, Tavassoli FA (2000) Concurrent and independent genetic alterations in the stromal and epithelial cells of mammary carcinoma: implications for tumorigenesis. Cancer Res 60:2562-2566

27. Kurose K, Gilley K, Matsumoto S, Watson PH, Zhou XP, Eng C (2002) Frequent somatic mutations in PTEN and TP53 are mutually exclusive in the stroma of breast carcinomas. Nat Genet 32:355-357

28. Kurose K, Hoshaw-Woodard S, Adeyinka A, Lemeshow S, Watson PH, Eng C (2001) Genetic model of multi-step breast carcinogenesis involving the epithelium and stroma: clues to tumour-microenvironment interactions. Hum Mol Genet 10:19071913

29. Kunz-Schughart LA, Knuechel R (2002) Tumor-associated fibroblasts (part I): Active stromal participants in tumor development and progression? Histol Histopathol 17:599-621

30. Kunz-Schughart LA, Knuechel R (2002) Tumor-associated fibroblasts (part II): Functional impact on tumor tissue. Histol Histopathol 17:623-637

31. Yu H, Maurer F, Medcalf RL (2002) Plasminogen activator inhibitor type 2: a regulator of monocyte proliferation and differentiation. Blood 99:2810-2818

32. Ranson M, Tian Z, Andronicos NM, Rizvi S, Allen BJ (2002) In vitro cytotoxicity of bismuth-213 (213Bi)-labeled-plasminogen activator inhibitor type 2 (alpha-PAI-2) on human breast cancer cells. Breast Cancer Res Treat 71:149-159

33. Allen BJ, Tian Z, Rizvi SM, Li Y, Ranson M (2003) Preclinical studies of targeted alpha therapy for breast cancer using 213Bilabelled-plasminogen activator inhibitor type 2. $\mathrm{Br} \mathrm{J}$ Cancer 88:944-950

34. Sieuwerts AM, Martens JW, Dorssers LC, Klijn JG, Foekens JA (2002) Differential effects of fibroblast growth factors on 
expression of genes of the plasminogen activator and insulin-like growth factor systems by human breast fibroblasts. Thromb Haemost 87:674-683

35. Fredriksson L, Li H, Fieber C, Li X, Eriksson U (2004) Tissue plasminogen activator is a potent activator of PDGF-CC. EMBO J 23:3793-3802

36. Torres-Collado AX, Kisiel W, Iruela-Arispe ML, RodriguezManzaneque JC (2006) ADAMTS1 interacts with, cleaves, and modifies the extracellular location of the matrix inhibitor tissue factor pathway inhibitor-2. J Biol Chem 281:17827-17837

37. Clavel C, Polette M, Doco M, Binninger I, Birembaut P (1992) Immunolocalization of matrix metallo-proteinases and their tissue inhibitor in human mammary pathology. Bull Cancer 79:261-270

38. Nguyen N, Kuliopulos A, Graham RA, Covic L (2006) Tumorderived Cyr61(CCN1) promotes stromal matrix metalloproteinase-1 production and protease-activated receptor 1-dependent migration of breast cancer cells. Cancer Res 66:2658-2665

39. Adolph KW (1999) Relative abundance of thrombospondin 2 and thrombospondin 3 mRNAs in human tissues. Biochem Biophys Res Commun 258:792-796

40. Esseghir S, Kennedy A, Seedhar P et al (2007) Identification of NTN4, TRA1, and STC2 as prognostic markers in breast cancer in a screen for signal sequence encoding proteins. Clin Cancer Res 13:3164-3173

41. Turashvili G, Bouchal J, Burkadze G, Kolar Z (2006) Wnt signaling pathway in mammary gland development and carcinogenesis. Pathobiology 73:213-223

42. Glinka A, Wu W, Delius H, Monaghan AP, Blumenstock C, Niehrs C (1998) Dickkopf-1 is a member of a new family of secreted proteins and functions in head induction. Nature 391:357-362

43. Voorzanger-Rousselot N, Goehrig D, Journe F et al (2007) Increased Dickkopf-1 expression in breast cancer bone metastases. Br J Cancer 97:964-970

44. Britsch S (2007) The neuregulin-I/ErbB signaling system in development and disease. Adv Anat Embryol Cell Biol 190:1-65

45. Krane IM, Leder P (1996) NDF/heregulin induces persistence of terminal end buds and adenocarcinomas in the mammary glands of transgenic mice. Oncogene 12:1781-1788

46. Sasano H, Suzuki T, Matsuzaki Y et al (1999) Messenger ribonucleic acid in situ hybridization analysis of estrogen receptors alpha and beta in human breast carcinoma. J Clin Endocrinol Metab 84:781-785

47. Palmieri C, Saji S, Sakaguchi H et al (2004) The expression of oestrogen receptor (ER)-beta and its variants, but not ERalpha, in adult human mammary fibroblasts. J Mol Endocrinol 33:35-50 